\title{
A Numerical Method for the Variable-Order Time-Fractional Wave Equations Based on the H2N2 Approximation
}

\author{
Xiao Liu $\mathbb{D}$, Yu Bo $\mathbb{D}$, and Yuanfeng Jin \\ College of Science, Yanbian University, Yanji 133002, China \\ Correspondence should be addressed to Yuanfeng Jin; yfkim@ybu.edu.cn
}

Received 1 October 2021; Revised 18 November 2021; Accepted 24 November 2021; Published 17 January 2022

Academic Editor: Youssri Hassan Youssri

Copyright (c) 2022 Xiao Liu et al. This is an open access article distributed under the Creative Commons Attribution License, which permits unrestricted use, distribution, and reproduction in any medium, provided the original work is properly cited.

\begin{abstract}
Aiming at the initial boundary value problem of variable-order time-fractional wave equations in one-dimensional space, a numerical method using second-order central difference in space and H2N2 approximation in time is proposed. A finite difference scheme with second-order accuracy in space and $3-\gamma^{*}$ order accuracy in time is obtained. The stability and convergence of the scheme are further discussed by using the discrete energy analysis method. A numerical example shows the effectiveness of the results.
\end{abstract}

\section{Introduction}

In recent years, due to the non-locality of fractional calculus, more and more problems in physical science, electromagnetism, electrochemistry, diffusion and general transport theory can be described by the fractional calculus approach, among which the Riemann-Liouville fractional derivative and the Caputo fractional derivative are the most widely used [1-4]. At the same time, more and more researchers found that a variety of important dynamical problems exhibit fractional-order behavior that may vary with time, space, or other conditions. This phenomenon indicates that variable-order fractional calculus is a natural choice to provide an effective mathematical framework for the description of complex problems.

In 2020, Shen et al. proposed a new numerical approximation method-the H2N2 approximation [5] for the numerical differential formula of the Caputo fractional derivative of $\gamma$ $\epsilon(1,2)$ and applied it for the constant-order time-fractional wave equations in the following multidimensional space

$$
\left\{\begin{array}{l}
{ }_{0}^{C} D_{t}^{\gamma} u(x, t)=\Delta u+q(x, t), x \in \Omega, t \in(0, T], \\
u(x, 0)=\varphi(x), u_{t}(x, 0)=\psi(x), x \in \Omega, \\
u(x, t)=0, \quad x \in \partial \Omega, t \in[0, T],
\end{array}\right.
$$

where $q(x, t), \varphi(x), \psi(x)$ are given sufficiently smooth functions, $\Omega=\prod_{j=1}^{d}\left(l^{(j)}, r^{(j)}\right) \subset R^{d}, \partial \Omega$ is the boundary of $\Omega, x=$ $\left(x^{(1)}, x^{(2)}, \cdots, x^{(d)}\right) \in \Omega, \Delta u=\sum_{j=1}^{d} \partial_{x^{(j)}}^{2} u$. When $x \in \partial \Omega, \varphi(x)$ and $\psi(x)$ satisfy consistency conditions $\varphi(x)=\psi(x)=0$. It was proved that the proposed scheme has the accuracy of order of $(3-\gamma)$ in time and 2 in space, and it is clear that its theoretical analysis is similar to the L1 method applied in solving the constant-order time-fractional slow diffusion equations.

Motivated by the above literature [6-9], in this work, we consider the numerical solution of the following variableorder time-fractional wave equations in one-dimensional space

$$
\begin{aligned}
{ }_{0}^{C} D_{t}^{\gamma(t)} u(x, t) & =u_{x x}(x, t)+f(x, t), x \in(0, L), t \in(0, T] . \\
u(x, 0) & =\varphi(x), u_{t}(x, 0)=\psi(x), x \in(0, L) . \\
u(0, t) & =0, u(L, t)=0, t \in[0, T] .
\end{aligned}
$$

where $1<\gamma(t)<2{ }_{0}^{C} D_{t}^{\gamma(t)} u(x, t)$ is the variable-order Caputo fractional derivative, $f(x, t), \varphi(x), \psi(x)$ are given suffificiently smooth functions and satisfy $\varphi(0)=\psi(0), \varphi(L)=\psi(L)$. Suppose its solution function $u \in C^{(4,3)}([0, L] \times[0, T])$. 
The rest of this paper is organized as follows. In the next section, some necessary notations are introduced. In Section 3 , the H2N2-based finite difference scheme for the variableorder time-fractional wave equations is derived. In Section 4, the stability and convergence of the difference scheme are studied. In Section 5, a numerical result is listed to verify the theoretical prediction and the effectiveness of the difference scheme. Finally, a brief conclusion is provided.

\section{Preliminary Knowledge and Relevant Lemmas}

Definition 1 (see [10]). Suppose the function $f(t)$ is defined on the interval $[0, T], 1<\gamma(t)<2$, then the variable-order Caputo fractional derivative is defined as

$$
{ }_{0}^{C} D_{t}^{\gamma(t)} f(t)=\frac{1}{\Gamma(2-\gamma(t))} \int_{0}^{t} f^{\prime \prime}(s)(t-s)^{1-\gamma(t)} d s .
$$

Next, mesh the solution intervals $[0, L]$ and $[0, T]$, take integers $M$ and $N$, denote $h=L / M, \tau=T / N, h$ and $\tau$ are called space step and time step, respectively. Denote $x_{i}=i h(0 \leq i \leq$ $M), t_{k}=k \tau(0 \leq k \leq N), \Omega_{h}=\left\{x_{i} \mid 0 \leq i \leq M\right\}, \Omega_{\tau}=\left\{t_{k} \mid 0 \leq k \leq\right.$ $N\}$. Define the following grid function spaces

$$
\begin{aligned}
& U_{h}=\left\{u \mid u=\left(u_{0}, u_{1}, \cdots, u_{M}\right)\right\}, \\
& \widehat{U}_{h}=\left\{u \mid u \in U_{h}, u_{0}=u_{M}=0\right\} .
\end{aligned}
$$

For grid function $u=\left\{u_{i}^{k} \mid 0 \leq i \leq M, 0 \leq k \leq N\right\}$ defined on $\Omega_{h} \times \Omega_{\tau}$, introduce the following notations

$$
\begin{aligned}
\delta_{x} u_{i-1 / 2}^{k} & =\frac{1}{h}\left(u_{i}^{k}-u_{i-1}^{k}\right), \\
\delta_{x}^{2} u_{i}^{k} & =\frac{1}{h^{2}}\left(u_{i+1}^{k}-2 u_{i}^{k}+u_{i-1}^{k}\right), \\
\delta_{t} u_{i}^{k+1 / 2} & =\frac{1}{\tau}\left(u_{i}^{k+1}-u_{i}^{k-1}\right), \\
\delta_{t}^{2} u_{i}^{k} & =\frac{1}{\tau}\left(\delta_{t} u_{i}^{k+1 / 2}-\delta_{t} u_{i}^{k-1 / 2}\right) .
\end{aligned}
$$

For any grid functions $u, v \in \widehat{U}_{h}$, denote the following notations

$$
\begin{aligned}
(u, v) & =h \sum_{i=1}^{M-1} u_{i} v_{i}, \quad\|u\|=\sqrt{(u, u)}, \\
\left(\delta_{x} u, \delta_{x} v\right) & =h \sum_{i=0}^{M-1}\left(\delta_{x} u_{i+1 / 2}\right)\left(\delta_{x} v_{i+1 / 2}\right), \\
\|u\|_{\infty} & =\max _{0 \leq i \leq M}\left|u_{i}\right|, \quad\left\|\delta_{x} u\right\|=\sqrt{\left(\delta_{x} u, \delta_{x} u\right)} .
\end{aligned}
$$

For any function $f(t)$ defined on the interval $\left[0, t_{1}\right]$, using the data $\left(t_{0}, f\left(t_{0}\right)\right),\left(t_{1}, f\left(t_{1}\right)\right),\left(t_{0}, f^{\prime}\left(t_{0}\right)\right)$ to make the quadratic Hermite interpolation polynomial of $f(t)$

$$
H_{2,0}(t)=f\left(t_{0}\right)+f^{\prime}\left(t_{0}\right)\left(t-t_{0}\right)+\frac{1}{\tau}\left(\delta_{t} f^{1 / 2}-f^{\prime}\left(t_{0}\right)\right)\left(t-t_{0}\right)^{2} .
$$

Taking the twice derivative arrives at

$$
H_{2,0}^{\prime}(t)=\frac{2}{\tau}\left(\delta_{t} f^{1 / 2}-f^{\prime}\left(t_{0}\right)\right) .
$$

For any function $f(t)$ defined on the interval $\left[t_{k-1}, t_{k+1}\right]$ $(1 \leq k \leq N-1)$, using three points $\left(t_{k-1}, f\left(t_{k-1}\right)\right),\left(t_{k}, f\left(t_{k}\right)\right)$, $\left(t_{k+1}, f\left(t_{k+1}\right)\right)$ to make the quadratic Newton interpolation polynomial of $f(t)$

$$
\begin{aligned}
N_{2, k}(t)= & f\left(t_{k-1}\right)+\left(\delta_{t} f^{k-1 / 2}\right)\left(t-t_{k-1}\right) \\
& +\frac{1}{2}\left(\delta_{t}^{2} f^{k}\right)\left(t-t_{k-1}\right)\left(t-t_{k}\right) .
\end{aligned}
$$

Taking the second-order derivative yields

$$
N_{2, k}^{\prime}(t)=\delta_{t}^{2} f^{k} .
$$

On the basis of the above interpolation polynomial, we next discuss the high-precision approximation formula of the variable-order Caputo fractional derivative.

Here, we denote $f^{l}=f\left(t_{l}\right), \gamma_{n-1 / 2}=\gamma\left(t_{n-1 / 2}\right), t_{n-1 / 2}=t_{n}$ $-\tau / 2$. Suppose $f(t) \in C^{3}\left[t_{0}, t_{n}\right]$ and $1<\gamma(t)<2$, then at the half-grid point $t_{n-1 / 2}$, we have

$$
\begin{aligned}
{ }_{0}^{C} D_{t}^{\gamma_{n-1 / 2}} f\left(t_{n-1 / 2}\right)= & \frac{1}{\Gamma\left(2-\gamma_{n-1 / 2}\right)}\left[\int_{t_{0}}^{t_{1 / 2}} f^{\prime \prime}(t)\left(t_{n-1 / 2}-t\right)^{1-\gamma_{n-1 / 2}} d t\right. \\
& \left.+\sum_{k=1}^{n-1} \int_{t_{k-1 / 2}}^{t_{k+1 / 2}} f^{\prime \prime}(t)\left(t_{n-1 / 2}-t\right)^{1-\gamma_{n-1 / 2}} d t\right] \\
\approx & \frac{1}{\Gamma\left(2-\gamma_{n-1 / 2}\right)}\left[\int_{t_{0}}^{t_{1 / 2}} H_{2,0}^{\prime}(t)\left(t_{n-1 / 2}-t\right)^{1-\gamma_{n-1 / 2}} d t\right. \\
& \left.+\sum_{k=1}^{n-1} \int_{t_{k-1 / 2}}^{t_{k+1 / 2}} N_{2, k}^{\prime}(t)\left(t_{n-1 / 2}-t\right)^{1-\gamma_{n-1 / 2}} d t\right]=\frac{1}{\Gamma\left(2-\gamma_{n-1 / 2}\right)} \\
& \cdot\left[\int_{t_{0}}^{t_{1 / 2}} \frac{2}{\tau}\left(\delta_{t} f^{1 / 2}-f^{\prime}\left(t_{0}\right)\right)\left(t_{n-1 / 2}-t\right)^{1-\gamma_{n-1 / 2}} d t\right. \\
& \left.+\sum_{k=1}^{n-1} \int_{t_{k-1 / 2}}^{t_{k+1 / 2}}\left(\delta_{t}^{2} f^{k}\right)\left(t_{n-1 / 2}-t\right)^{1-\gamma_{n-1 / 2}} d t\right]=\frac{1}{\Gamma\left(2-\gamma_{n-1 / 2}\right)} \\
& \cdot\left[\frac{2}{\tau} \int_{t_{0}}^{t_{1 / 2}}\left(t_{n-1 / 2}-t\right)^{1-\gamma_{n-1 / 2}} d t \cdot\left(\delta_{t} f^{1 / 2}-f^{\prime}\left(t_{0}\right)\right)\right. \\
& \left.+\frac{1}{\tau} \sum_{k=1}^{n-1} \int_{t_{k-1 / 2}}^{t_{k+1 / 2}}\left(t_{n-1 / 2}-t\right)^{1-\gamma_{n-1 / 2} d t} \cdot\left(\delta_{t} f^{k+1 / 2}-\delta_{t} f^{k-1 / 2}\right)\right] \\
= & \frac{1}{\Gamma\left(2-\gamma_{n-1 / 2}\right)}\left[b_{n-1}^{\left(n, \gamma_{n-1 / 1}\right)}\left(\delta_{t} f^{1 / 2}-f^{\prime}\left(t_{0}\right)\right)+\sum_{k=1}^{n-1} b_{n-k-1}^{\left(n, \gamma_{n-1 / 2}\right.}\right) \\
& \left.\cdot\left(\delta_{t} f^{k+1 / 2}-\delta_{t} f^{k-1 / 2}\right)\right]=\frac{1}{\Gamma\left(2-\gamma_{n-1 / 2}\right)} \\
& \cdot\left[b_{0}^{\left(n, \gamma_{n-1 / 2}\right)} \delta_{t} f^{n-1 / 2}-\sum_{k=1}^{n-1}\left(b_{n-k-1}^{\left(n, \gamma_{n-1 / 2}\right)}-b_{n-k}^{\left(n, \gamma_{n-1 / 2}\right)}\right) \delta_{t} f^{k-1 / 2}\right. \\
& \left.-b_{n-1}^{\left(n, \gamma_{n-1 / 2}\right)} f^{\prime}\left(t_{0}\right)\right] \equiv D_{t}^{\gamma_{n-1 / 2}} f\left(t_{n-1 / 2}\right) . \\
&
\end{aligned}
$$


Here

$$
\begin{aligned}
& b_{n-1}^{\left(n, \gamma_{n-1 / 2}\right)}=\frac{2}{\tau} \int_{t_{0}}^{t_{1 / 2}}\left(t_{n-1 / 2}-t\right)^{1-\gamma_{n-1 / 2}} d t, \\
& b_{n-k-1}^{\left(n, \gamma_{n-1 / 2}\right)}=\frac{1}{\tau} \int_{t_{k-1 / 2}}^{t_{k+1 / 2}}\left(t_{n-1 / 2}-t\right)^{1-\gamma_{n-1 / 2}} d t,
\end{aligned}
$$

where $1 \leq k \leq n-1$.

Then, it can be calculated that

$$
b_{k}^{\left(n, \gamma_{n-1 / 2}\right)}= \begin{cases}\frac{\tau^{1-\gamma_{n-1 / 2}}}{2-\gamma_{n-1 / 2}}\left[(k+1)^{\left.2-\gamma_{n-1 / 2}-k^{2-\gamma_{n-1 / 2}}\right],}\right. & 0 \leq k \leq n-2, \\ 2 \frac{\tau^{1-\gamma_{n-1 / 2}}}{2-\gamma_{n-1 / 2}}\left[\left(n-\frac{1}{2}\right)^{2-\gamma_{n-1 / 2}}-(n-1)^{2-\gamma_{n-1 / 2}}\right], & k=n-1 .\end{cases}
$$

Denote

$$
r_{n}={ }_{0}^{C} D_{t}^{\gamma_{n-1 / 2}} f\left(t_{n-1 / 2}\right)-D_{t}^{\gamma_{n-1 / 2}} f\left(t_{n-1 / 2}\right),
$$

we have

$$
\left|r_{n}\right| \leq C_{0_{0} \leq t \leq t_{n}} \max \left|f^{\prime \prime \prime}(t)\right| \tau^{3-\gamma_{n-1 / 2}}
$$

Here, $C_{0}=1 / 8 \Gamma\left(2-\gamma_{n-1 / 2}\right)+1 / 12 \Gamma\left(3-\gamma_{n-1 / 2}\right)+\left(\gamma_{n-1 / 2}\right.$ $-1) / 2 \Gamma\left(4-\gamma_{n-1 / 2}\right)$, the proof process is similar to Theorem 2.1 in Reference [5].

Lemma 2. For any $n \geq 2$, according to $b_{k}^{\left(n, \gamma_{n-1 / 2}\right)}$ defined by (14)-(15), we have

$$
\begin{aligned}
\frac{\tau^{1-\gamma_{n-1 / 2}}}{(n-1 / 2)^{\gamma_{n-1 / 2}-1}} & <b_{n-1}^{\left(n, \gamma_{n-1 / 2}\right)}<b_{n-2}^{\left(n, \gamma_{n-1 / 2}\right)}<\cdots<b_{1}^{\left(n, \gamma_{n-1 / 2}\right)} \\
& <b_{0}^{\left(n, \gamma_{n-1 / 2}\right)}=\frac{\tau^{1-\gamma_{n-1 / 2}}}{2-\gamma_{n-1 / 2}} .
\end{aligned}
$$

Proof. According to the formula (14)-(15), we have

$$
b_{n-1}^{\left(n, \gamma_{n-1 / 2}\right)}=\frac{2 \tau^{1-\gamma_{n-1 / 2}}}{2-\gamma_{n-1 / 2}}\left[\left(n-\frac{1}{2}\right)^{2-\gamma_{n-1 / 2}}-(n-1)^{2-\gamma_{n-1 / 2}}\right],
$$

$b_{n-k-1}^{\left(n, \gamma_{n-1 / 2}\right)}=\frac{\tau^{1-\gamma_{n-1 / 2}}}{2-\gamma_{n-1 / 2}}\left[(n-k)^{2-\gamma_{n-1 / 2}}-(n-k-1)^{2-\gamma_{n-1 / 2}}\right], \quad 1 \leq k \leq n-1$.

When $k=n-1$, it can be obtained by calculation

$$
b_{0}^{\left(n, \gamma_{n-1 / 2}\right)}=\frac{\tau^{1-\gamma_{n-1 / 2}}}{2-\gamma_{n-1 / 2}} .
$$

From equations (20) and (21), we have

$$
\begin{aligned}
b_{n-1}^{\left(n, \gamma_{n-1 / 2}\right)} & =2 \tau^{1-\gamma_{n-1 / 2}} \int_{n-1}^{n-1 / 2} \xi^{1-\gamma_{n-1 / 2}} d \xi, \\
b_{k}^{\left(n, \gamma_{n-1 / 2}\right)} & =\tau^{1-\gamma_{n-1 / 2}} \int_{k}^{k+1} \xi^{1-\gamma_{n-1 / 2}} d \xi, \\
0 & \leq k \leq n-2 .
\end{aligned}
$$

Therefore, it can be obtained

$$
b_{n-1}^{\left(n, \gamma_{n-1 / 2}\right)}<b_{n-2}^{\left(n, \gamma_{n-1 / 2}\right)}<\cdots<b_{1}^{\left(n, \gamma_{n-1 / 2}\right)}<b_{0}^{\left(n, \gamma_{n-1 / 2}\right)}=\frac{\tau^{1-\gamma_{n-1 / 2}}}{2-\gamma_{n-1 / 2}} .
$$

When $n \geq 2$, we have

$$
\begin{aligned}
\left(1-\frac{1}{2 n-1}\right)^{2-\gamma_{n-1 / 2}}= & 1-\frac{2-\gamma_{n-1 / 2}}{2 n-1} \\
& +\frac{\left(2-\gamma_{n-1 / 2}\right)\left(1-\gamma_{n-1 / 2}\right)}{2 !}\left(-\frac{1}{2 n-1}\right)^{2} \\
& +\frac{\left(2-\gamma_{n-1 / 2}\right)\left(1-\gamma_{n-1 / 2}\right)\left(-\gamma_{n-1 / 2}\right)}{3 !} \\
& \cdot\left(-\frac{1}{2 n-1}\right)^{3}+\cdots
\end{aligned}
$$

From the above formula

$$
\begin{aligned}
(n- & \left.\frac{1}{2}\right)^{2-\gamma_{n-1 / 2}}-(n-1)^{2-\gamma_{n-1 / 2}}-\frac{2-\gamma_{n-1 / 2}}{2(n-1 / 2)^{\gamma^{n-1 / 2-1}}} \\
= & \left(n-\frac{1}{2}\right)^{2-\gamma_{n-1 / 2}}\left[1-\frac{2-\gamma_{n-1 / 2}}{2(n-1 / 2)}-\left(1-\frac{1}{2 n-1}\right)^{2-\gamma_{n-1 / 2}}\right] \\
= & \left(n-\frac{1}{2}\right)^{2-\gamma_{n-1 / 2}}\left[-\frac{\left(2-\gamma_{n-1 / 2}\right)\left(1-\gamma_{n-1 / 2}\right)}{2 !}\left(-\frac{1}{2 n-1}\right)^{2}\right. \\
& \left.-\frac{\left(2-\gamma_{n-1 / 2}\right)\left(1-\gamma_{n-1 / 2}\right)\left(-\gamma_{n-1 / 2}\right)}{3 !}\left(-\frac{1}{2 n-1}\right)^{3}-\cdots\right]>0 .
\end{aligned}
$$

And when $n=1$, we have

$$
\left(\frac{1}{2}\right)^{2-\gamma_{n-1 / 2}}-\frac{2-\gamma_{n-1 / 2}}{2 \cdot(1 / 2)^{\gamma_{n-1 / 2}-1}}=\frac{\gamma_{n-1 / 2}-1}{2^{2-\gamma_{n-1 / 2}}}>0 .
$$

Therefore, it can be seen that

$$
b_{n-1}^{\left(n, \gamma_{n-1 / 2}\right)}>\frac{2 \tau^{1-\gamma_{n-1 / 2}}}{2-\gamma_{n-1 / 2}} \cdot \frac{2-\gamma_{n-1 / 2}}{2(n-1 / 2)^{\gamma_{n-1 / 2}-1}}=\frac{\tau^{1-\gamma_{n-1 / 2}}}{(n-1 / 2)^{\gamma_{n-1 / 2}-1}} .
$$

To sum up, Lemma 2 is proved. 
Lemma 3 (see [11]). If the function $f \in C^{4}\left[x_{i-1}, x_{i+1}\right], \lambda \in($ $\left.x_{i-1}, x_{i+1}\right)$, there is

$$
f^{\prime \prime}\left(x_{i}\right)=\frac{f\left(x_{i-1}\right)-2 f\left(x_{i}\right)+f\left(x_{i+1}\right)}{h^{2}}-\frac{h^{2}}{12} f^{(4)}(\lambda) .
$$

Lemma 4. For any positive integer $m$ and any $\psi, V_{1}, V_{2}, \cdots$, $V_{N} \in \widehat{U}_{h}$, when

$$
\left(t_{n+1 / 2}-t\right)^{\gamma_{n+1 / 2}-1} \geq\left(t_{n-1 / 2}-t\right)^{\gamma_{n-1 / 2}-1}, t \in\left(0, t_{n-1 / 2}\right), t_{n+1 / 2} \leq T,
$$

we have

$$
\begin{aligned}
& \sum_{n=1}^{m}\left(b_{0}^{\left(n, \gamma_{n-1 / 2}\right)} V^{n}-\sum_{k=1}^{n-1}\left(b_{n-k-1}^{\left(n, \gamma_{n-1 / 2}\right)}-b_{n-k}^{\left(n, \gamma_{n-1 / 2}\right)}\right) V^{k}-b_{n-1}^{\left(n, \gamma_{n-1 / 2}\right)} \psi, V^{n}\right) \\
& \quad \geq \frac{1}{2}\left(\sum_{k=1}^{m} b_{m-k}^{\left(m, \gamma_{m-1 / 2}\right)}\left\|V^{k}\right\|^{2}-\sum_{n=1}^{m} b_{n-1}^{\left(n, \gamma_{n-1 / 2}\right)}\|\psi\|^{2}\right),
\end{aligned}
$$

where $1 \leq m \leq N$.

Proof. On the basis of [12], it can be seen from the condition

$$
\begin{aligned}
& \sum_{n=1}^{m}\left(b_{0}^{\left(n, \gamma_{n-1 / 2}\right)} V^{n}-\sum_{k=1}^{n-1}\left(b_{n-k-1}^{\left(n, \gamma_{n-1 / 2}\right)}-b_{n-k}^{\left(n, \gamma_{n-1 / 2}\right)}\right) V^{k}-b_{n-1}^{\left(n, \gamma_{n-1 / 2}\right)} \psi, V^{n}\right) \\
& =\sum_{n=1}^{m}\left(b_{0}^{\left(n, \gamma_{n-1 / 2}\right)}\left\|V^{n}\right\|^{2}-\sum_{k=1}^{n-1}\left(b_{n-k-1}^{\left(n, \gamma_{n-1 / 2}\right)}-b_{n-k}^{\left(n, \gamma_{n-1 / 2}\right)}\right)\left(V^{k}, V^{n}\right)\right. \\
& \left.-b_{n-1}^{\left(n, \gamma_{n-1 / 2}\right)}\left(\psi, V^{n}\right)\right) \geq \sum_{n=1}^{m}\left[b_{0}^{\left(n, \gamma_{n-1 / 2}\right)}\left\|V^{n}\right\|^{2}-\frac{1}{2} \sum_{k=1}^{n-1}\right. \\
& \left.\cdot\left(b_{n-k-1}^{\left(n, \gamma_{n-1 / 2}\right)}-b_{n-k}^{\left(n, \gamma_{n-1 / 2}\right)}\right)\left(\left\|V^{k}\right\|^{2}+\left\|V^{n}\right\|^{2}\right)-\frac{1}{2} b_{n-1}^{\left(n, \gamma_{n-1 / 2}\right)}\left(\|\psi\|^{2}+\left\|V^{n}\right\|^{2}\right)\right] \\
& =\frac{1}{2} \sum_{n=1}^{m}\left[\left(2 b_{0}^{\left(n, \gamma_{n-1 / 2}\right)}-\sum_{k=1}^{n-1}\left(b_{n-k-1}^{\left(n, \gamma_{n-1 / 2}\right)}-b_{n-k}^{\left(n, \gamma_{n-1 / 2}\right)}\right)-b_{n-1}^{\left(n, \gamma_{n-1 / 2}\right)}\right)\left\|V^{n}\right\|^{2}\right. \\
& \left.-\sum_{k=1}^{n-1}\left(b_{n-k-1}^{\left(n, \gamma_{n-1 / 2}\right)}-b_{n-k}^{\left(n, \gamma_{n-1 / 2}\right)}\right)\left\|V^{k}\right\|^{2}-b_{n-1}^{\left(n, \gamma_{n-1 / 2}\right)}\|\psi\|^{2}\right] \\
& =\frac{1}{2} \sum_{n=1}^{m}\left[b_{0}^{\left(n, \gamma_{n-1 / 2}\right)}\left\|V^{n}\right\|^{2}-\sum_{k=1}^{n-1} b_{n-k-1}^{\left(n, \gamma_{n-1 / 2}\right)}\left\|V^{k}\right\|^{2}+\sum_{k=1}^{n-1} b_{n-k}^{\left(n, \gamma_{n-1 / 2}\right)}\left\|V^{k}\right\|^{2}\right. \\
& \left.-b_{n-1}^{\left(n, \gamma_{n-1 / 2}\right)}\|\psi\|^{2}\right]=\frac{1}{2} \sum_{n=1}^{m}\left[\sum_{k=1}^{n} b_{n-k}^{\left(n, \gamma_{n-1 / 2}\right)}\left\|V^{k}\right\|^{2}-\sum_{k=1}^{n-1} b_{n-k-1}^{\left(n, \gamma_{n-1 / 2}\right)}\left\|V^{k}\right\|^{2}\right. \\
& \left.-b_{n-1}^{\left(n, \gamma_{n-1 / 2}\right)}\|\psi\|^{2}\right]=\frac{1}{2}\left[\sum_{k=1}^{m} \sum_{n=k}^{m} b_{n-k}^{\left(n, \gamma_{n-1 / 2}\right)}\left\|V^{k}\right\|^{2}-\sum_{k=1}^{m-1} \sum_{n=k+1}^{m} b_{n-k-1}^{\left(n, \gamma_{n-1 / 2}\right)}\left\|V^{k}\right\|^{2}\right. \\
& \left.-\sum_{n=1}^{m} b_{n-1}^{\left(n, \gamma_{n-1 / 2}\right)}\|\psi\|^{2}\right]=\frac{1}{2}\left[b_{0}^{\left(m, \gamma_{m-1 / 2}\right)}\left\|V^{m}\right\|^{2}\right. \\
& \left.+\sum_{k=1}^{m-1}\left(\sum_{n=k}^{m} b_{n-k}^{\left(n, \gamma_{n-1 / 2}\right)}-\sum_{n=k+1}^{m} b_{n-k-1}^{\left(n, \gamma_{n-1 / 2}\right)}\right)\left\|V^{k}\right\|^{2}-\sum_{n=1}^{m} b_{n-1}^{\left(n, \gamma_{n-1 / 2}\right)}\|\psi\|^{2}\right] \\
& =\frac{1}{2}\left[b_{0}^{\left(m, \gamma_{m-1 / 2}\right)}\left\|V^{m}\right\|^{2}+\sum_{k=1}^{m-1} b_{m-k}^{\left(m, \gamma_{m-1 / 2}\right)}\left\|V^{k}\right\|^{2}\right. \\
& \left.+\sum_{k=1}^{m-1} \sum_{n=k}^{m-1}\left(b_{n-k}^{\left(n, \gamma_{n-1 / 2}\right)}-b_{n-k}^{\left(n+1, \gamma_{n+1 / 2}\right)}\right)\left\|V^{k}\right\|^{2}-\sum_{n=1}^{m} b_{n-1}^{\left(n, \gamma_{n-1 / 2}\right)}\|\psi\|^{2}\right] .
\end{aligned}
$$

When $\gamma(t)$ satisfies the following condition

$$
\begin{aligned}
& b_{n-k}^{\left(n, \gamma_{n-1 / 2}\right)}-b_{n-k}^{\left(n+1, \gamma_{n+1 / 2}\right)} \\
& \quad=\frac{1}{\tau} \int_{t_{k-3 / 2}}^{t_{k-1 / 2}}\left[\left(t_{n-1 / 2}-t\right)^{1-\gamma_{n-1 / 2}}-\left(t_{n+1 / 2}-t\right)^{1-\gamma_{n+1 / 2}}\right] d t,
\end{aligned}
$$

namely

$$
\left(t_{n+1 / 2}-t\right)^{\gamma_{n+1 / 2}-1} \geq\left(t_{n-1 / 2}-t\right)^{\gamma_{n-1 / 2}-1}
$$

Then, we have

$$
\begin{aligned}
& \sum_{n=1}^{m}\left(b_{0}^{\left(n, \gamma_{n-1 / 2}\right)} V^{n}-\sum_{k=1}^{n-1}\left(b_{n-k-1}^{\left(n, \gamma_{n-1 / 2}\right)}-b_{n-k}^{\left(n, \gamma_{n-1 / 2}\right)}\right) \cdot V^{k}-b_{n-1}^{\left(n, \gamma_{n-1 / 2}\right)} \psi, V^{n}\right) \\
& \quad \geq \frac{1}{2}\left(\sum_{k=1}^{m} b_{m-k}^{\left(m, \gamma_{m-1 / 2}\right)}\left\|V^{k}\right\|^{2}-\sum_{n=1}^{m} b_{n-1}^{\left(n, \gamma_{n-1 / 2}\right)}\|\psi\|^{2}\right) .
\end{aligned}
$$

Remark 5. Consider the function

$$
g(x, y)=x^{y}, \quad x>0, y>0
$$

We have

$$
\begin{aligned}
& \frac{\partial g(x, y)}{\partial x}=y x^{y-1}>0 \\
& \frac{\partial g(x, y)}{\partial y}=x^{y} \operatorname{In} x= \begin{cases}<0, & x \in(0,1) \\
>0, & x>1\end{cases}
\end{aligned}
$$

If $T \leq 1$ and $\gamma(t)$ is an non-increasing function on $[0, T]$, then $t_{n+1 / 2}-t \in(0,1), t_{n-1 / 2}-t \in(0,1)$ and $\gamma_{n+1 / 2} \leq \gamma_{n-1 / 2}$, consequently

$\left(t_{n+1 / 2}-t\right)^{\gamma_{n+1 / 2}-1} \geq\left(t_{n-1 / 2}-t\right)^{\gamma_{n-1 / 2}-1}, t \in\left(0, t_{n-1 / 2}\right), t_{n+1 / 2} \leq T$.

(30) is valid.

If $T>1, \gamma(t)$ is an non-increasing function on the interval $[0,1]$ and $\gamma(t)$ is a constant on the interval $[1, T],(30)$ is also valid.

Lemma 6 (see [11]). For any $\varepsilon>0, a, b \geq 0$, there is

$$
a b \leq \varepsilon a^{2}+\frac{1}{4 \varepsilon} b^{2} .
$$

Lemma 7 (see [11]). For any grid function $u \in \widehat{U}_{h}$, there is

$$
\|u\|_{\infty} \leq \frac{\sqrt{L}}{2}\left\|\delta_{x} u\right\|
$$


Lemma 8 (see [13]). Suppose $\left\{F^{k} \mid k \geq 0\right\},\left\{G^{k} \mid k \geq 1\right\}$ are two non-negative sequences, $\left\{G^{k}\right\}$ does not decrease with $k$, if

$$
F^{k} \leq C \tau \sum_{l=0}^{k} F^{l}+G^{k}, \quad k=1,2, \cdots
$$

where $C$ is an non-negative constant, when $\tau \leq 2 / 3 C$, then

$$
F^{k} \leq \exp (3 C k \tau)\left(C \tau F^{0}+3 G^{k}\right), \quad k=1,2, \cdots
$$

\section{Establishment of the Difference Scheme}

Denote $U_{i}^{n}=u\left(x_{i}, t_{n}\right), 0 \leq i \leq M, 0 \leq n \leq N ; \varphi_{i}=\varphi\left(x_{i}\right), \psi_{i}=$ $\psi\left(x_{i}\right)$, consider (2) at the point $\left(x_{i}, t_{n-1 / 2}\right)$, we have

$$
\begin{aligned}
{ }_{0}^{C} D_{t}^{\gamma_{n-1 / 2}} u\left(x_{i}, t_{n-1 / 2}\right) & =u_{x x}\left(x_{i}, t_{n-1 / 2}\right)+f\left(x_{i}, t_{n-1 / 2}\right), \quad 1 \leq i \\
& \leq M-1,1 \leq n \leq N .
\end{aligned}
$$

Applying (13) to approximate the temporal fractional derivative and central difference quotient (29) to approximate the spatial derivative, we can obtain

$$
\begin{aligned}
& \frac{1}{\Gamma\left(2-\gamma_{n-1 / 2}\right)}\left[b_{0}^{\left(n, \gamma_{n-1 / 2}\right)} \delta_{t} U_{i}^{n-1 / 2}-\sum_{k=1}^{n-1}\right. \\
& \left.\quad \cdot\left(b_{n-k-1}^{\left(n, \gamma_{n-1 / 2}\right)}-b_{n-k}^{\left(n, \gamma_{n-1 / 2}\right)}\right) \delta_{t} U_{i}^{k-1 / 2}-b_{n-1}^{\left(n, \gamma_{n-1 / 2}\right)} \psi_{i}\right] \\
& =\delta_{x}^{2} U_{i}^{n-1 / 2}+f_{i}^{n-1 / 2}+R_{i}^{n-1 / 2}, \quad 1 \leq i \leq M-1,1 \leq n \leq N .
\end{aligned}
$$

There exists a positive constant $C_{1}$ such that

$$
\left|R_{i}^{n-1 / 2}\right| \leq C_{1}\left(\tau^{3-\gamma_{n-1 / 2}}+h^{2}\right), \quad 1 \leq i \leq M-1,1 \leq n \leq N .
$$

Noticing the initial and boundary value conditions (3) and (4), we have

$$
\left\{\begin{array}{l}
U_{i}^{0}=\varphi_{i}, \quad 1 \leq i \leq M-1 . \\
U_{0}^{n}=0, \quad U_{M}^{n}=0, \quad 0 \leq n \leq N .
\end{array}\right.
$$

Omitting the small term $R_{i}^{n-1 / 2}$ in the equation and replacing the grid function $U_{i}^{n}$ by its numerical approximation $u_{i}^{n}$, we construct the difference scheme for solving the problems (2)-(4) as follows

$$
\begin{gathered}
\frac{1}{\Gamma\left(2-\gamma_{n-1 / 2}\right)}\left[b_{0}^{\left(n, \gamma_{n-1 / 2}\right)} \delta_{t} u_{i}^{n-1 / 2}-\sum_{k=1}^{n-1}\right. \\
\left.\cdot\left(b_{n-k-1}^{\left(n, \gamma_{n-1 / 2}\right)}-b_{n-k}^{\left(n, \gamma_{n-1 / 2}\right)}\right) \delta_{t} u_{i}^{k-1 / 2}-b_{n-1}^{\left(n, \gamma_{n-1 / 2}\right)} \psi_{i}\right] \\
=\delta_{x}^{2} u_{i}^{n-1 / 2}+f_{i}^{n-1 / 2}, \quad 1 \leq i \leq M-1,1 \leq n \leq N .
\end{gathered}
$$

$$
\begin{aligned}
& u_{i}^{0}=\varphi_{i}, \quad 1 \leq i \leq M-1 . \\
& u_{0}^{n}=0, \quad u_{M}^{n}=0,0 \leq n \leq N .
\end{aligned}
$$

\section{Stability and Convergence of the Difference Scheme}

Theorem 9. Suppose $\left\{u_{i}^{n} \mid 0 \leq i \leq M, 0 \leq n \leq N\right\}$ is the solution of the following difference scheme

$$
\begin{gathered}
\frac{1}{\Gamma\left(2-\gamma_{n-1 / 2}\right)}\left[b_{0}^{\left(n, \gamma_{n-1 / 2}\right)} \delta_{t} u_{i}^{n-1 / 2}-\sum_{k=1}^{n-1}\right. \\
\left.\cdot\left(b_{n-k-1}^{\left(n, \gamma_{n-1 / 2}\right)}-b_{n-k}^{\left(n, \gamma_{n-1 / 2}\right)}\right) \delta_{t} u_{i}^{k-1 / 2}-b_{n-1}^{\left(n, \gamma_{n-1 / 2}\right)} \psi_{i}\right] \\
=\delta_{x}^{2} u_{i}^{n-1 / 2}+p_{i}^{n-1 / 2}, \quad 1 \leq i \leq M-1,1 \leq n \leq N, \\
u_{i}^{0}=\varphi_{i}, \quad 1 \leq i \leq M-1, \\
u_{0}^{n}=0, \quad u_{M}^{n}=0,0 \leq n \leq N,
\end{gathered}
$$

where $p_{i}^{n-1 / 2}$ is a given perturbation term, when $\tau<2 / 3 c_{0}$, it holds that

$$
\left\|\delta_{x} u^{n}\right\|^{2} \leq \exp \left(3 c_{0} T\right)\left(c_{0} \tau\left\|\delta_{x} u^{0}\right\|^{2}+3 Q^{n}\right), \quad 1 \leq n \leq N .
$$

$c_{0}$ and $Q^{n}$ are given in (56) and (64), respectively.

Proof. Taking an inner product (50) with $\Gamma\left(2-\gamma_{n-1 / 2}\right) \delta_{t}$ $u^{n-1 / 2}$ and summing $n$ from 1 to $m$, we have

$$
\begin{aligned}
& \sum_{n=1}^{m}\left[b_{0}^{\left(n, \gamma_{n-1 / 2}\right)}\left\|\delta_{t} u^{n-1 / 2}\right\|^{2}-\sum_{k=1}^{n-1}\left(b_{n-k-1}^{\left(n, \gamma_{n-1 / 2}\right)}-b_{n-k}^{\left(n-\gamma_{n-1 / 2}\right)}\right)\left(\delta_{t} u^{k-1 / 2}, \delta_{t} u^{n-1 / 2}\right)\right. \\
& \left.\quad-b_{n-1}^{\left(n, \gamma_{n-1 / 1}\right)}\left(\psi, \delta_{t} u^{n-1 / 2}\right)\right]=\sum_{n=1}^{m} \Gamma\left(2-\gamma_{n-1 / 2}\right)\left(\delta_{x}^{2} u^{n-1 / 2}, \delta_{t} u^{n-1 / 2}\right) \\
& \quad+\sum_{n=1}^{m} \Gamma\left(2-\gamma_{n-1 / 2}\right)\left(p^{n-1 / 2}, \delta_{t} u^{n-1 / 2}\right), \quad 1 \leq m \leq N .
\end{aligned}
$$


Noticing that

$$
\begin{aligned}
& \sum_{n=1}^{m} \Gamma\left(2-\gamma_{n-1 / 2}\right)\left(\delta_{x}^{2} u^{n-1 / 2}, \delta_{t} u^{n-1 / 2}\right) \\
&=- \frac{1}{2 \tau} \sum_{n=1}^{m} \Gamma\left(2-\gamma_{n-1 / 2}\right)\left(\left\|\delta_{x} u^{n}\right\|^{2}-\left\|\delta_{x} u^{n-1}\right\|^{2}\right) \\
&=-\frac{1}{2 \tau} \sum_{n=1}^{m}\left\{\Gamma\left(2-\gamma_{n}\right)\left\|\delta_{x} u^{n}\right\|^{2}-\Gamma\left(2-\gamma_{n-1}\right)\left\|\delta_{x} u^{n-1}\right\|^{2}\right. \\
&-\left[\Gamma\left(2-\gamma_{n}\right)-\Gamma\left(2-\gamma_{n-1 / 2}\right)\right]\left\|\delta_{x} u^{n}\right\|^{2}-\left[\Gamma\left(2-\gamma_{n-1 / 2}\right)\right. \\
&\left.\left.-\Gamma\left(2-\gamma_{n-1}\right)\right]\left\|\delta_{x} u^{n-1}\right\|^{2}\right\} \leq-\frac{1}{2 \tau} \sum_{n=1}^{m} \\
& \cdot\left(\Gamma\left(2-\gamma_{n}\right)\left\|\delta_{x} u^{n}\right\|^{2}-\Gamma\left(2-\gamma_{n-1}\right)\left\|\delta_{x} u^{n-1}\right\|^{2}\right) \\
&+\frac{1}{2 \tau} \sum_{n=1}^{m}\left[\Gamma\left(2-\gamma_{n}\right)-\Gamma\left(2-\gamma_{n-1 / 2}\right)\right]\left\|\delta_{x} u^{n}\right\|^{2} \\
&+\frac{1}{2 \tau} \sum_{n=1}^{m}\left[\Gamma\left(2-\gamma_{n-1 / 2}\right)-\Gamma\left(2-\gamma_{n-1}\right)\right]\left\|\delta_{x} u^{n-1}\right\|^{2} \\
& \leq-\frac{1}{2 \tau} \sum_{n=1}^{m}\left(\Gamma\left(2-\gamma_{n}\right)\left\|\delta_{x} u^{n}\right\|^{2}-\Gamma\left(2-\gamma_{n-1}\right)\left\|\delta_{x} u^{n-1}\right\|^{2}\right) \\
&+\frac{1}{4} c_{0} \sum_{n=1}^{m}\left(\left\|\delta_{x} u^{n}\right\|^{2}+\left\|\delta_{x} u^{n-1}\right\|^{2}\right)
\end{aligned}
$$

where

$$
c_{0}=\max _{0 \leq t \leq T}\left|\frac{d}{d t} \Gamma(2-\gamma(t))\right| \text {. }
$$

Applying Lemma 4, we have

$$
\begin{aligned}
& \frac{1}{2}\left(\sum_{k=1}^{m} b_{m-k}^{\left(m, \gamma_{m-1 / 2}\right)}\left\|\delta_{t} u^{k-1 / 2}\right\|^{2}-\sum_{n=1}^{m} b_{n-1}^{\left(n, \gamma_{n-1 / 2}\right)}\|\psi\|^{2}\right) \\
& \leq-\frac{1}{2 \tau} \sum_{n=1}^{m}\left(\Gamma\left(2-\gamma_{n}\right)\left\|\delta_{x} u^{n}\right\|^{2}-\Gamma\left(2-\gamma_{n-1}\right)\left\|\delta_{x} u^{n-1}\right\|^{2}\right) \\
& \quad+\frac{1}{4} c_{0} \sum_{n=1}^{m}\left(\left\|\delta_{x} u^{n}\right\|^{2}+\left\|\delta_{x} u^{n-1}\right\|^{2}\right)+\sum_{n=1}^{m} \Gamma\left(2-\gamma_{n-1 / 2}\right) \\
& \quad \times\left(p^{n-1 / 2}, \delta_{t} u^{n-1 / 2}\right), \quad 1 \leq m \leq N .
\end{aligned}
$$

Then, we have

$$
\begin{gathered}
\frac{1}{2} \sum_{k=1}^{m} b_{m-k}^{\left(m, \gamma_{m-1 / 2}\right)}\left\|\delta_{t} u^{k-1 / 2}\right\|^{2}+\frac{1}{2 \tau}\left(\Gamma\left(2-\gamma_{m}\right)\left\|\delta_{x} u^{m}\right\|^{2}-\left\|\delta_{x} u^{0}\right\|^{2}\right) \\
\leq \frac{1}{2} \sum_{n=1}^{m} b_{n-1}^{\left(n, \gamma_{n-1 / 2}\right)}\|\psi\|^{2}+\sum_{n=1}^{m} \Gamma\left(2-\gamma_{n-1 / 2}\right)\left(p^{n-1 / 2}, \delta_{t} u^{n-1 / 2}\right) \\
+\frac{1}{2} c_{0} \sum_{n=0}^{m}\left\|\delta_{x} u^{n}\right\|^{2}, \quad 1 \leq m \leq N .
\end{gathered}
$$

$$
\begin{aligned}
\frac{1}{2} t_{m-1 / 2}^{1-\gamma_{m-1 / 2}} \sum_{k=1}^{m}\left\|\delta_{t} u^{k-1 / 2}\right\|^{2}+\frac{\Gamma\left(2-\gamma_{m}\right)}{2 \tau}\left\|\delta_{x} u^{m}\right\|^{2} \\
\leq \frac{1}{2 \tau}\left\|\delta_{x} u^{0}\right\|^{2}+\frac{1}{2} \sum_{n=1}^{m} \frac{\tau^{1-\gamma_{n-1 / 2}}}{2-\gamma_{n-1 / 2}}\|\psi\|^{2}+\sum_{n=1}^{m} \Gamma\left(2-\gamma_{n-1 / 2}\right) \\
\cdot\left(\frac{t_{m-1 / 2}^{1-\gamma_{m-1 / 2}}}{2 \Gamma\left(2-\gamma_{n-1 / 2}\right)}\left\|\delta_{t} u^{n-1 / 2}\right\|^{2}+\frac{\Gamma\left(2-\gamma_{n-1 / 2}\right)}{2 t_{m-1 / 2}^{1-\gamma_{m-1 / 2}}}\left\|p^{n-1 / 2}\right\|^{2}\right) \\
+\frac{1}{2} c_{0} \sum_{n=0}^{m}\left\|\delta_{x} u^{n}\right\|^{2} .
\end{aligned}
$$

We use the Cauchy inequality for the inner product $\left(p^{n-1 / 2}, \delta_{t} u^{n-1 / 2}\right)$, the above equation can be simplified

$$
\begin{aligned}
\frac{\Gamma\left(2-\gamma_{m}\right)}{2 \tau}\left\|\delta_{x} u^{m}\right\|^{2} \leq & \frac{1}{2 \tau}\left\|\delta_{x} u^{0}\right\|^{2}+\frac{1}{2} \sum_{n=1}^{m} \frac{\tau^{1-\gamma_{n-1 / 2}}}{2-\gamma_{n-1 / 2}}\|\psi\|^{2} \\
& +\sum_{n=1}^{m} \frac{\Gamma^{2}\left(2-\gamma_{n-1 / 2}\right)}{2 t_{m-1 / 2}^{1-\gamma_{m-1 / 2}}}\left\|p^{n-1 / 2}\right\|^{2} \\
& +\frac{1}{2} c_{0} \sum_{n=0}^{m}\left\|\delta_{x} u^{n}\right\|^{2} .
\end{aligned}
$$

Multiplying by $2 \tau / \Gamma\left(2-\gamma_{m}\right)$, then we have

$$
\begin{aligned}
\left\|\delta_{x} u^{m}\right\|^{2} \leq & \frac{1}{\Gamma\left(2-\gamma_{m}\right)}\left(\left\|\delta_{x} u^{0}\right\|^{2}+\tau \sum_{n=1}^{m} \frac{\tau^{1-\gamma_{n-1 / 2}}}{2-\gamma_{n-1 / 2}}\|\psi\|^{2}\right. \\
& \left.+\tau t_{m-1 / 2}^{\gamma_{m-1 / 2}-1} \sum_{n=1}^{m} \Gamma^{2}\left(2-\gamma_{n-1 / 2}\right)\left\|p^{n-1 / 2}\right\|^{2}+\tau c_{0} \sum_{n=0}^{m}\left\|\delta_{x} u^{n}\right\|^{2}\right) .
\end{aligned}
$$

Note that $\Gamma$ is decreasing on the interval $(0,1]$. Since 0 $<2-\gamma(t) \leq 1, \Gamma(2-\gamma(t))^{-1} \leq 1$. Then

$$
\begin{aligned}
\left\|\delta_{x} u^{m}\right\|^{2} \leq & \left\|\delta_{x} u^{0}\right\|^{2}+\tau \sum_{n=1}^{m} \frac{\tau^{1-\gamma_{n-1 / 2}}}{2-\gamma_{n-1 / 2}}\|\psi\|^{2} \\
& +\tau t_{m-1 / 2}^{\gamma_{m-1 / 2}-1} \sum_{n=1}^{m} \Gamma^{2}\left(2-\gamma_{n-1 / 2}\right)\left\|p^{n-1 / 2}\right\|^{2} \\
& +\tau c_{0} \sum_{n=0}^{m}\left\|\delta_{x} u^{n}\right\|^{2} .
\end{aligned}
$$


Let

$$
\begin{aligned}
Q^{m}= & \left\|\delta_{x} u^{0}\right\|^{2}+\tau \sum_{n=1}^{m} \frac{\tau^{1-\gamma_{n-1 / 2}}}{2-\gamma_{n-1 / 2}}\|\psi\|^{2}+\tau t_{m-1 / 2}^{\gamma_{m-1 / 2}-1} \sum_{n=1}^{m} \Gamma^{2} \\
& \cdot\left(2-\gamma_{n-1 / 2}\right)\left\|p^{n-1 / 2}\right\|^{2}, \quad 1 \leq m \leq N .
\end{aligned}
$$

Then

$$
\left\|\delta_{x} u^{m}\right\|^{2} \leq \tau c_{0} \sum_{n=0}^{m}\left\|\delta_{x} u^{n}\right\|^{2}+Q^{m}
$$

It is easy to know $Q^{m}$ does not decrease with $m$. According to Lemma 8 , when $\tau<2 / 3 c_{0}$, we have

$$
\left\|\delta_{x} u^{m}\right\|^{2} \leq \exp \left(3 c_{0} T\right)\left(c_{0} \tau\left\|\delta_{x} u^{0}\right\|^{2}+3 Q^{m}\right), \quad 1 \leq m \leq N .
$$

Theorem 9 is proved. We can say that the difference scheme is stable.

Theorem 10. Assume $\left\{u\left(x_{i}, t_{n}\right)\right\}$ and $\left\{u_{i}^{n}\right\}$ are solutions of problems (2)-(4) and difference scheme (47)-(49), respectively. Denote

$$
e_{i}^{n}=u\left(x_{i}, t_{n}\right)-u_{i}^{n}, \quad 0 \leq i \leq M, \quad 0 \leq n \leq N .
$$

Then, there exists a positive constant $C_{2}$, when $\tau<2 / 3 c_{0}$,
TABLE 1: Errors and temporal convergence orders, $M=1000$.

\begin{tabular}{cccc}
\hline$\gamma(t)$ & $\tau$ & $E(h, \tau)$ & Order $_{\tau}$ \\
\hline \multirow{4}{*}{$2-t^{2}$} & $1 / 512$ & $8.7295 e-4$ & 0 \\
& $1 / 1024$ & $4.2265 e-4$ & 1.05 \\
& $1 / 2048$ & $2.0502 e-4$ & 1.04 \\
& $1 / 4096$ & $9.9746 e-5$ & 1.04 \\
\hline \multirow{3}{*}{$1+e^{-t}$} & $1 / 512$ & $4.0772 e-4$ & 0 \\
& $1 / 1024$ & $1.8602 e-4$ & 1.13 \\
& $1 / 2048$ & $8.5528 e-5$ & 1.12 \\
& $1 / 4096$ & $3.9764 e-5$ & 1.10 \\
\hline \multirow{3}{*}{$\frac{\cos t}{4}$} & $1 / 512$ & $3.8173 e-4$ & 0 \\
& $1 / 1024$ & $1.5945 e-4$ & 1.26 \\
& $1 / 2048$ & $6.6746 e-5$ & 1.26 \\
& $1 / 4096$ & $2.8186 e-5$ & 1.24 \\
\hline
\end{tabular}

$n \tau \leq T$, such that

$$
\left\|e^{n}\right\|_{\infty} \leq \frac{\sqrt{L}}{2} C_{2}\left(\tau^{3-\gamma^{*}}+h^{2}\right), \quad 0 \leq n \leq N,
$$

where $C_{2}=\sqrt{3 T L} C_{1} \exp \left(3 c_{0} T / 2\right)$ and $\gamma^{*}=\max _{0 \leq t \leq T} \gamma(t)$.

Proof. Subtracting (44) and (46) from (47)-(49), we obtain the system of error equations

$$
\left\{\begin{array}{l}
\frac{1}{\Gamma\left(2-\gamma_{n-1 / 2}\right)}\left[b_{0}^{\left(n, \gamma_{n-1 / 2}\right)} \delta_{t} e_{i}^{n-1 / 2}-\sum_{k=1}^{n-1}\left(b_{n-k-1}^{\left(n, \gamma_{n-1 / 2}\right)}-b_{n-k}^{\left(n, \gamma_{n-1 / 2}\right)}\right) \delta_{t} e_{i}^{k-1 / 2}\right]=\delta_{x}^{2} e_{i}^{n-1 / 2}+R_{i}^{n-1 / 2}, \\
1 \leq i \leq M-1, \quad 1 \leq n \leq N, \\
e_{i}^{0}=0, \quad 1 \leq i \leq M-1, \\
e_{0}^{n}=0, \quad e_{M}^{n}=0, \quad 0 \leq n \leq N .
\end{array}\right.
$$

Applying Theorem 9 and (45), it yields

$$
\left\|\delta_{x} e^{n}\right\|^{2} \leq C_{2}^{2}\left(\tau^{3-\gamma^{*}}+h^{2}\right)^{2}
$$

where $\gamma^{*}=\max _{0 \leq t \leq T} \gamma(t)$.

Applying Lemma 7, it yields

$$
\left\|e^{n}\right\|_{\infty} \leq \frac{\sqrt{L}}{2}\left\|\delta_{x} e^{n}\right\| \leq \frac{\sqrt{L}}{2} C_{2}\left(\tau^{3-\gamma^{*}}+h^{2}\right) .
$$

The proof is ended.

\section{Numerical Example}

In order to verify the accuracy of the finite difference scheme, several different types of variable-order index $\gamma(t)$ $\epsilon(1,2)$ are used to solve the variable-order fractional wave equations (2)-(4) in $1 \mathrm{D}$ case. The scheme is implemented in MATLAB (R2019a).

Here, we take $L=\pi, T=1$. The source term of equation (2)

$f(x, t)=\left(\frac{6}{\Gamma(4-\gamma(t))} t^{3-\gamma(t)}+\frac{6}{\Gamma(3-\gamma(t))} t^{2-\gamma(t)}+t^{3}+3 t^{2}+1\right) \sin x$, 
TABLe 2: Errors and spatial convergence orders, $N=20000$.

\begin{tabular}{cccc}
\hline$\gamma(t)$ & $h$ & $E(h, \tau)$ & Order $_{h}$ \\
\hline \multirow{3}{*}{$2-t^{2}$} & $\pi / 5$ & $3.2641 e-2$ & 0 \\
& $\pi / 10$ & $8.6512 e-3$ & 1.92 \\
& $\pi / 20$ & $2.1801 e-3$ & 1.99 \\
& $\pi / 40$ & $5.5869 e-4$ & 1.96 \\
\hline \multirow{2}{*}{$1+e^{-t}$} & $\pi / 5$ & $3.1901 e-2$ & 0 \\
& $\pi / 10$ & $8.4489 e-3$ & 1.92 \\
& $\pi / 20$ & $2.1209 e-3$ & 1.99 \\
$6+\cos t$ & $\pi / 40$ & $5.3548 e-4$ & 1.99 \\
\hline 4 & $\pi / 5$ & $3.0356 e-2$ & 0 \\
& $\pi / 10$ & $8.0401 e-3$ & 1.92 \\
& $\pi / 20$ & $2.0164 e-3$ & 2.00 \\
& $\pi / 40$ & $5.0707 e-4$ & 1.99 \\
\hline
\end{tabular}

the initial value

$$
u(x, 0)=\sin x, \quad x \in[0, \pi],
$$

the boundary value

$$
u(0, t)=u(\pi, t)=0, \quad t \in[0,1],
$$

the exact solution is given by

$$
u(x, t)=\left(t^{3}+3 t^{2}+1\right) \sin x .
$$

Define the error of the numerical solution

$$
E(h, \tau)=\max _{0 \leq k \leq N}\left\|U^{k}-u^{k}\right\|_{\infty},
$$

the temporal convergence order

$$
\operatorname{Order}_{\tau}=\log _{2}\left(\frac{E(h, 2 \tau)}{E(h, \tau)}\right)
$$

the spatial convergence order

$$
\operatorname{Order}_{h}=\log _{2}\left(\frac{E(2 h, \tau)}{E(h, \tau)}\right) .
$$

Denote $M=1000$, for different $\gamma(t)=2-t^{2}, 1+e^{-t}$, (6 + cost)/4. The time step $\tau$ is varied from $1 / 512$ to $1 /$ 4096, where $N=512,1024,2048,4096$. Table 1 shows the errors and temporal convergence orders of the difference scheme (47)-(49). It can be seen from Table 1 that the difference scheme (47)-(49) has a precision of approximately $3-\gamma^{*}$ order in time. The computational results are in good agreement with theoretical results.

Take a fixed and sufficiently small time step $\tau=1 / 20000$, for different $\gamma(t)=2-t^{2}, 1+e^{-t},(6+\cos t) / 4$, verify space step $h$ from $\pi / 5$ to $\pi / 40$, where $N=20000, M=5,10,20,40$ . Table 2 shows the errors and spatial convergence orders of the difference scheme (47)-(49). It can be seen from Table 2 that the difference scheme (47)-(49) in the space has an accuracy of approximately 2 order, which is consistent with the theoretical results.

\section{Conclusions}

In this paper, we consider a numerical approximation method for the variable-order Caputo fractional derivative- $\mathrm{H} 2 \mathrm{~N} 2$ approximation, and give the corresponding calculation formula. Secondly, we use this formula to solve the one-dimensional variable-order time-fractional wave equations and discuss the stability and convergence of the equations by the discrete energy analysis method. Finally, a numerical example verifies the effectiveness of the scheme.

\section{Data Availability}

The data used to support the findings of this study are included within the article.

\section{Conflicts of Interest}

The authors declare that they have no conflicts of interest.

\section{Acknowledgments}

The work is supported by the National Natural Science Foundation of China (11761074), the Natural Science Foundation of Jilin Province (2020122336JC), and the Program for Young and Middle-aged Leading Talents in Scientific and Technological Innovation of Jilin Province (20200301053RQ).

\section{References}

[1] X. Zhao, Z. Z. Sun, and G. E. Karniadakis, "Second-order approximations for variable order fractional derivatives: Algorithms and applications," Journal of Computational Physics, vol. 293, pp. 184-200, 2015.

[2] Z. W. Fang, H. W. Sun, and H. Wang, "A fast method for variable-order Caputo fractional derivative with applications to time-fractional diffusion equations," Computers and Mathematics with Applications, vol. 80, no. 5, pp. 1443-1458, 2020.

[3] S. Chen, F. Liu, P. Zhuang, and V. Anh, "Finite difference approximations for the fractional Fokker-Planck equation," Applied Mathematical Modelling, vol. 33, no. 1, pp. 256-273, 2009.

[4] J. W. Guo, The Research on the Asymptotic Expansions of Solutions to Two Kinds of Variable-Order Fractional Equations and Related Numerical Algorithms, Tianjin Normal University, 2020.

[5] J. Y. Shen, C. P. Li, and Z. Z. Sun, “An H2N2 interpolation for Caputo derivative with order in $(1,2)$ and its application to time-fractional wave equations in more than one space dimension," Journal of Scientific Computing, vol. 83, no. 2, pp. 1-29, 2020.

[6] R. L. Du, A. A. Alikhanov, and Z. Z. Sun, "Temporal second order difference schemes for the multi-dimensional variableorder time fractional sub-diffusion equations," Computers 
Mathematics with Applications, vol. 79, no. 10, pp. 2952-2972, 2020.

[7] H. F. Ding, "The development of higher-order numerical differential formulas of Caputo derivative and their applications (I)," Computers and Mathematics with Applications, vol. 84, pp. 203-223, 2021.

[8] Y. F. Liu, A Compact Finite Difference Scheme for Variable Order Time Diffusion Equation, Shandong University, 2018.

[9] X. M. Ma, Finite Difference/Spectral Methods for VariableOrder Fractional Diffusion Equations, Xiamen University, 2017.

[10] H. G. Sun, W. Chen, H. Wei, and Y. Q. Chen, “A comparative study of constant-order and variable-order fractional models in characterizing memory property of systems," The European Physical Journal Special Topics, vol. 193, no. 1, pp. 185-192, 2011.

[11] Z. Z. Sun, Numerical Methods for Partial Differential Equations, Science Press, Beijing, 2012.

[12] Z. Z. Sun, C. C. Ji, and R. L. Du, "A new analytical technique of the L-type difference schemes for time fractional mixed subdiffusion and diffusion-wave equations," Applied Mathematics Letters, vol. 102, 2020.

[13] R. Liu, Finite Difference Methods for Solving A Class of MultiTerm Time Fractional Diffusion-Wave Equations with the Spatial Fourth-Order Derivative, Nanjing University of Posts and Telecommunications, 2018. 